\title{
The Cavity as a Lasting Abode for Tuberculous Bacilli
}

\section{An Observational Study}

\author{
Nadia Jawad ${ }^{1,2} \cdot$ Saira Jafri ${ }^{1,2} \cdot$ Nausheen Saifullah ${ }^{1,2} \cdot$ Naseem Ahmed $^{1,2}$ \\ Accepted: 10 December 2021 / Published online: 17 January 2022 \\ (c) The Author(s), under exclusive licence to Springer Nature Switzerland AG 2021
}

\begin{abstract}
The aim of this study was to determine the factors associated with persistent sputum positivity at the end of 2 months of treatment in patients presenting with (drug-susceptible) pulmonary tuberculosis at a tertiary care hospital in Karachi. A cross-sectional study was conducted at the Department of Chest Medicine (Ward 12), Jinnah Postgraduate Medical Center (JPMC), Karachi, over 6 months. A sample of 73 consenting, newly diagnosed, smear-positive drug-susceptible pulmonary tuberculosis patients was studied. Demographic (age, gender, height, weight and duration of tuberculosis, BMI, socioeconomic, occupational, marital, educational and residential statuses) and clinical factors (chest X-ray extent and cavities, initial smear results, diabetic and smoking statuses) which may be associated with sputum non-conversion were entered in a proforma. Patients were followed up at 2 months of treatment with a sputum smear. Data analysis was done on SPSS (Statistical Package for Social Sciences-version 20.0). Rate of sputum positivity after 2 months of anti-tubercular drug treatment was $17.8 \%$. None of the above-mentioned demographic and clinical factors was associated with persistence of sputum positivity except for the presence of CXR cavities, which made it 5.5 times more likely that the patient would remain smear-positive at 2 months $(p=0.035)$. The finding of chest radiograph cavities makes it highly likely that a pulmonary tuberculosis patient may remain infectious or have an unfavorable outcome despite taking treatment for 2 months. Clinicians and national policymakers should thus bear in mind the implications this can have with regard to disease control and therefore pay particular attention to such patients in terms of stringent monitoring and directly observed treatment short-course (DOTS) provision.
\end{abstract}

Keywords Clinical factors $\cdot$ Drug-sensitive tuberculosis $\cdot$ Persistent positivity $\cdot$ Pulmonary tuberculosis $\cdot$ Smear-positive . Sputum smear

\section{Abbreviations}

AFB Acid-fast bacill

BMI Body mass index

CI Confidence interval

COVID-19 Coronavirus disease due to SARS CoV-2

CXR

DM

ESR

HIV

IFN

Diabetes mellitus

Erythrocyte sedimentation rate

Human immunodeficiency virus

Interferon

This article is part of the Topical Collection on Medicine

Saira Jafri

meetsaira@gmail.com

Karachi, Pakistan

2 Jinnah Postgraduate Medical Centre, Karachi, Pakistan

$\begin{array}{ll}\text { MDR } & \text { Multidrug resistant } \\ \text { OR } & \text { Odds ratio } \\ \text { PTB } & \text { Pulmonary tuberculosis } \\ \text { SPSS } & \text { Statistical Package for Social Sciences } \\ \text { TB } & \text { Tuberculosis } \\ \text { TTD } & \text { Time to detection } \\ \text { WHO } & \text { World Health Organization } \\ \text { XDR } & \text { Extensively drug resistant }\end{array}$

\section{Introduction}

Tuberculosis (TB) continues to be a colossal public health challenge globally and remains one of the world's deadliest diseases, killing about 1.5 million and infecting about 10 million people worldwide in 2018 [1]. An untreated patient has the potential to infect another 10-15 people each year, depending upon bacillary load, duration of close contact, 
and duration of antitubercular treatment [2,3]. On starting effective antitubercular treatment, bacillary load decreases rapidly, which correlate with reduced infectivity [4]. Therefore, infection control measures are recommended for all sputum smear-positive patients until serial smears convert to negative $[5,6]$.

Sputum microscopy for acid-fast bacilli serves as a wellvalidated and cost-effective means of measuring treatment response [7]. Non-conversion of sputum smear at the end of the second month of treatment has been documented to be associated with unfavorable outcomes such as failure and relapse within 2 years of follow-up [8,9]. Several factors that correlate with heavy initial bacillary load (higher sputum smear and culture grading at diagnosis, shorter time to detection (TTD) of Mycobacterium tuberculosis on liquid cultures) and radiologically extensive disease have been linked with sputum smear/culture non-conversion at the end of the second month of TB treatment [10]. Additionally, increasing age, erythrocyte sedimentation rate (ESR), male sex, smoking, diabetes mellitus (DM), malnutrition, anemia, and thrombocytosis have also been associated with persistent sputum positivity [11, 12].

Through this study, we sought to determine the factors associated with persistent sputum positivity at the end of 2 months of treatment in patients with drug-sensitive pulmonary tuberculosis presenting to a tertiary care hospital in the metropolitan city of Karachi.

In the COVID era, co-infection with COVID-19 raises concerns about the interactions between the two diseases with respect to immune responses, morbidity, and mortality. SARS CoV-2 activates the adaptive immune response, with the $\mathrm{CD} 4+\mathrm{T}$ cell response providing protective immunity [13]. In a patient with latent/active TB, co-infection may alter the course of COVID-19 disease. Though little is known about the exact immune responses at play in coinfected patients, evidence provided by a study performed on whole blood and bronchoalveolar lavage fluid suggest that active TB increases the risk of severe COVID-19 disease, due to increased abundance of circulating myeloid subpopulations and increased interferon (IFN) production [14].

The first ever global cohort of COVID-19 and active/ former tuberculosis patients constituted 49 patients ( $42 \mathrm{had}$ active TB and 7 had TB sequelae) recruited by the Global Tuberculosis Network (GTN) in 8 countries on 3 continents [15]. This observational study concluded that COVID-19 could occur before, after, or simultaneously with TB. No causal associations were established due to the small number of patients. Also, authors agreed that larger, longitudinal studies were required to definitively ascertain the role of COVID-19 in accelerating progression from latent to active TB and the impact of TB sequelae on COVID-19 outcomes.

The global TB and COVID-19 study by the GTN/WHO on nearly 700 patients in 40 countries is expected to clarify many ambiguities on the interactions of the two diseases, including the risk of progression to active TB in those with latent TB [16].

TB/COVID-19 co-infection may spell poor outcomes, including higher mortality. Combined data from the 8 countries and Sondalo Hospital study show an $11.6 \%$ case fatality rate (8/69) [17]. The study shows that mortality may be more related to occurrence of comorbidities and older age than $\mathrm{TB}$ alone. However, higher death rates may occur in cases with resistant or severe forms of TB even in the young.

The COVID-19 pandemic has given rise to new fears about the fate of undiagnosed/unreported TB patients as access to TB care services has become limited. This is evidenced by a drop in TB notification rates [18]. High burden countries like Pakistan need to step up policies and invest in active case-finding strategies so that the success gained in terms TB mortality is not reversed.

Since our study was predominantly conducted before the pandemic took hold in Pakistan, we did not consider or test for COVID-19 among our enrolled patients.

Through this study, we sought to determine the factors associated with persistent sputum positivity at the end of 2 months of treatment in patients with drug-sensitive pulmonary tuberculosis presenting to a tertiary care hospital in the metropolitan city of Karachi.

\section{Material and Methods}

\section{Study Design, Setting, and Duration}

This cross-sectional study was conducted after an ethical approval (No. F.2-81/2019-GENL/21153/JPMC) at the Department of Chest Medicine (Ward 12), Jinnah Postgraduate Medical Center (JPMC), Karachi, over a period of about 6 months from September 14, 2019, to March 16, 2020.

\section{Patients}

Consenting adult patients aged 20-60 years of either gender (randomly selected among out-patients) with pulmonary infiltrate (opacity with or without air bronchogram) or cavity on chest radiograph along with persistent productive cough and fever (body temperature $\geq 37.8^{\circ} \mathrm{C}$ ) and confirmed by positive sputum smear on sputum microscopy for acid-fast bacilli (AFB) were included and labeled as having pulmonary tuberculosis. Patients who did not give consent and had history of hepatitis C and B or human immunodeficiency virus (HIV) infection, malignancy, sarcoidosis, TB treatment failure, TB treatment interruption, retreatment, or multi/ extensively drug-resistant (MDR/XDR) TB were excluded. Patients with malignancy, HIV, and hepatitis B or C were 
excluded as the occurrence of the above conditions may alter the presentation of TB disease due to immunosuppression.

\section{Sampling}

Assuming the proportion of smear grade $+1,19.6 \%$ [6], based on 95\% two-sided significance level (1-alpha), $80 \%$ power, and $9 \%$ margin of error, the representative sample size calculated (considering the local study by D'Souza et al.) was 73 patients using non-probability consecutive sampling.

\section{Board Approval}

This study was conducted after approval (No. F.2-81/2019GENL/21153/JPMC) from the institutional ethical review board. Informed consent was obtained from all the patients for assigning them to the study and using their data in research.

\section{Procedures and Investigations}

A brief history of demographic data, duration of disease, comorbids, and smoking habits was taken and recorded in a form. A person who smoked at least 100 cigarettes in his/ her lifetime was labelled a smoker (according to Centers for Disease Control and Prevention https://www.cdc.gov/nchs/ nhis/tobacco/tobacco_glossary.htm). All those who were known diabetics and on anti-diabetic treatment were considered as diabetics.

Chest X-ray findings (with regard to extent and cavitation) were also noted at diagnosis. Each participant's height (in meters) and weight (to the nearest kilogram) were measured. Every patient received weight-based anti-tubercular drug regimens (comprising isoniazid, rifampicin, ethambutol, and pyrazinamide) as recommended by the National TB Control Programme of Pakistan [19] (based on WHO Guidelines for treatment of drug-susceptible tuberculosis and patient care, 2017 update [20]). Drug compliance was evaluated (on compliance form) by confirmation from close relative of patient and retrieval of empty blister packs of prescribed drugs.

Patients' sputum was examined with microscopy and Xpert MTB/RIF (to rule out rifampicin resistance) before initiating treatment and with microscopy at 2 months of treatment. Sputum induction was done by means of $3 \%$ saline nebulization for patients who were unable to spontaneously produce sputum. The smears were prepared by the Ziehl-Neelsen method, examined for AFB by microscopy and graded according to the number of acid-fast bacilli (AFB) observed per high power field (HPF) as follows [21]:
- $>10$ AFB per oil immersion field in 20 fields as $3+$ positive

- 1-10 AFB per oil immersion field in 50 fields as $2+$ positive

- 10-99 AFB per 100 oil immersion fields in 100 fields as $1+$ positive

- 1-9 AFB per 100 oil immersion fields in 100 fields as sputum positive scanty

\section{Data Analysis}

Data were analyzed on SPSS (Statistical Package for Social Sciences) version 20.0. Mean and standard deviations were calculated for the quantitative variables like age, height, weight, and duration of tuberculosis. Frequencies and percentages were calculated for the qualitative variables like gender, BMI, socioeconomic status, occupational status, marital status, educational status, residential status, and clinical factors such as results of initial smear, chest X-ray extension, presence of chest X-ray cavities, smoking, and diabetes mellitus. Effect modifiers were controlled through stratification of age, gender, BMI, socioeconomic status, occupational status, marital status, and educational status to see the effect of these on outcome (smear positivity). Post stratification chi-square test was applied on stratified variables as well as the above-mentioned clinical factors associated with the persistent sputum positivity by taking $p$-value of $\leq 0.050$ as statistically significant. Odds ratio was also calculated, and $\mathrm{OR}>1$ was considered significant.

\section{Results}

Males comprised the majority of the 73 patients $(n=43,58.9 \%)$. Overall mean age of the patients was $38.6 \pm 15.6$ years. Less than half the participants $(n=30$, $41.1 \%$ ) had a normal BMI, with more than a third being underweight (37\%). Most (58.9\%) patients were literate to some extent, and all 73 patients belonged to lower to middle socioeconomic strata, which reflects the socioeconomic status of most, if not all, patients who visit public sector hospitals. Smokers constituted $61.6 \%(n=45)$ of the study participants, whereas diabetics made up three-quarters $(n=56,76.9 \%)$.

On initial sputum analysis, smear grade $3+$ was the most common $(n=30,41.1 \%)$, followed by $2+(n=19)$, scanty $(n=15)$, and $1+(n=9)$. A substantial number of patients had unilateral (61.6\%), multilobar (60.3\%) involvement on chest X-ray, while more than half $(n=41,56.2 \%)$ had one or more cavities.

After 2 months of initiation of antitubercular drugs, 60 $(82.2 \%)$ had negative follow-up sputum smears, whereas 13 
$(17.8 \%)$ were found to be sputum smear positive, of which 5 $(6.8 \%)$ were scanty, $6(8.2 \%)$ were $1+$, and $2(2.7)$ were $2+$.

None of the demographic features (gender, age, BMI, occupation, education, marital status, and socio-economic status) was found to be significantly associated with persistent sputum positivity (Table 1). The proportion of smokers was less among non-converted as compared with converted group (30.8\% vs. $40.0 \%, p=0.754)$, though not statistically significant (Table 2). Similarly, the differences with regard to diabetes and the extent of radiologic involvement between both groups were statistically unremarkable. Initial bacillary load as indicated by smear grade and Xpert burden did not predict sputum non-conversion $(p=0.460$ and 0.566 , respectively).

However, $84.6 \%$ of the non-converted patients had one or more cavities on chest radiograph (CXR) $(p=0.031)$, while half the patients who converted had cavities. CXR cavitation was found in most patients with high initial smear grades, precisely $68.4 \%$ of those with grade $2+$ and $60 \%$ of those with grade $3+$, but this was statistically insignificant $(p=0.330)$.

Further analysis by logistic regression revealed that CXR cavities were 5.5 times more likely to be associated with persistence of AFB on follow-up smears $(\mathrm{OR}=5.5)$ as shown in Table 3.

\section{Discussion}

The proportion of patients whose sputum did not convert in our study was $17.8 \%$, similar to other studies from the region (11.1\% by Wang et al [22], $18 \%$ by Sheetal et al [5], $19.5 \%$ by D'Souza et al [6]). A few studies have shown relatively higher rates of non-conversion $(25.3 \%$ by Diktanas et al [23], 33.2\% by Yellappa et al [24], 25.4\% by Motao et al [25]).

All of the demographic variables including age, gender, and body mass index (BMI) were found to be non-significant in relation to smear positivity which is similar to the study by Bouti et al [10], who reported no association of age, sex, weight, alcoholism, addictions, and previous TB disease. In contrast, some studies have cited increasing age and/or male gender as predictors of sputum nonconversion [3, 6, 11, 23-25].

The presence of diabetes or smoking did not impact the rate of conversion at 2 months in the present study, a finding alike to many studies [10, 11, 23]. However, works by Babalik et al [12] and Sheetal et al [5] have shown that smokers are more likely to have delayed sputum conversion.

A multitude of studies [3, 6, 10, 22-26] have demonstrated the relationship between a high initial bacillary
Table 1 Association of sputum positivity with demographic characteristics

\begin{tabular}{|c|c|c|c|c|c|}
\hline Factors & & Total & $\begin{array}{l}\text { Sputum con- } \\
\text { verted }(n=60)\end{array}$ & $\begin{array}{l}\text { Sputum non-con- } \\
\text { verted }(n=13)\end{array}$ & Sig. ${ }^{1}$ \\
\hline \multirow[t]{2}{*}{ Gender } & Male & $43(58.9)$ & $36(60.0)$ & $7(53.8)$ & 0.683 \\
\hline & Female & $30(41.1)$ & $24(40.0)$ & $6(46.2)$ & \\
\hline \multirow[t]{2}{*}{ Age (years) } & Below 40 & $38(52.1)$ & $32(53.3)$ & $6(46.2)$ & 0.639 \\
\hline & 40 or above & $35(47.9)$ & $28(46.7)$ & $7(53.8)$ & \\
\hline \multirow[t]{3}{*}{ BMI $\left(\mathrm{kg} / \mathrm{m}^{2}\right)^{2}$} & $<18.5$ (underweight) & $27(37.0)$ & $22(37.3)$ & $5(38.5)$ & 0.989 \\
\hline & $18.5-24.9$ (normal) & $30(41.1)$ & $24(40.7)$ & $5(38.5)$ & \\
\hline & $\geq 25$ (overweight) & $16(21.9)$ & $13(22.0)$ & $3(23.0)$ & \\
\hline \multirow[t]{3}{*}{ Occupation } & Student & $26(35.6)$ & $22(36.7)$ & $4(30.8)$ & 0.410 \\
\hline & Employed & $28(38.4)$ & $21(35.0)$ & $7(53.8)$ & \\
\hline & Unemployed & $19(26.0)$ & $17(28.3)$ & $2(15.4)$ & \\
\hline \multirow[t]{4}{*}{ Education } & Illiterate & $30(41.1)$ & $24(40.0)$ & $6(46.2)$ & 0.411 \\
\hline & Primary & $14(19.2)$ & $12(20.0)$ & $2(15.4)$ & \\
\hline & Secondary & $20(27.4)$ & $15(25.0)$ & $5(38.5)$ & \\
\hline & Higher & $9(12.3)$ & $9(15.0)$ & $0(0)$ & \\
\hline \multirow[t]{2}{*}{ Marital status } & Single & $23(31.5)$ & $20(33.3)$ & $3(23.1)$ & 0.743 \\
\hline & Married & $50(68.5)$ & $40(66.7)$ & $10(76.9)$ & \\
\hline \multirow[t]{3}{*}{ Socio-economic status } & Lower class & $32(43.8)$ & $26(43.3)$ & $6(46.2)$ & 0.885 \\
\hline & Lower middle class & $32(43.8)$ & $27(45.0)$ & $5(38.5)$ & \\
\hline & Middle class & $9(12.3)$ & 7 (11.7) & $2(15.4)$ & \\
\hline
\end{tabular}

Values given in parentheses are percentages

${ }^{1}$ Sig significance

${ }^{2} B M I$ body mass index 
Table 2 Association of sputum positivity with environmental and clinical factors

\begin{tabular}{|c|c|c|c|c|c|}
\hline Factors & & Total & $\begin{array}{l}\text { Sputum con- } \\
\text { verted }(n=60)\end{array}$ & $\begin{array}{l}\text { Sputum non-con- } \\
\text { verted }(n=13)\end{array}$ & Sig. $^{1}$ \\
\hline \multirow[t]{2}{*}{ House } & Own & $43(58.9)$ & $35(58.3)$ & $8(61.5)$ & 0.831 \\
\hline & Rented & $30(41.1)$ & $25(41.7)$ & $5(38.5)$ & \\
\hline \multirow[t]{2}{*}{ Smoker } & Yes & $45(61.6)$ & $24(40.0)$ & $4(30.8)$ & 0.754 \\
\hline & No & $28(38.4)$ & $36(60.0)$ & $9(69.2)$ & \\
\hline \multirow[t]{2}{*}{ Diabetic } & Yes & $56(76.7)$ & $14(23.3)$ & $3(23.1)$ & 0.984 \\
\hline & No & $17(23.3)$ & 46 (76.7) & $10(76.9)$ & \\
\hline \multirow[t]{4}{*}{ Initial smear } & Scanty & $15(20.5)$ & $14(23.3))$ & $1(7.7)$ & 0.460 \\
\hline & $1+$ & $9(12.3)$ & $8(13.3)$ & $1(7.7)$ & \\
\hline & $2+$ & $19(26.0)$ & $14(23.3)$ & $5(38.5)$ & \\
\hline & $3+$ & $30(41.1)$ & $24(40.0)$ & $6(46.2)$ & \\
\hline \multirow[t]{4}{*}{ Initial gen expert } & Very low/trace & $1(1.4)$ & $1(1.7)$ & $0(0)$ & 0.566 \\
\hline & Low & $15(20.5)$ & $14(23.3)$ & $1(7.7)$ & \\
\hline & Medium & $26(35.6)$ & $20(33.3)$ & $6(46.2)$ & \\
\hline & High & $31(42.5)$ & $25(41.7)$ & $6(46.2)$ & \\
\hline \multirow[t]{2}{*}{$\mathrm{CXR}^{2}$ extension side } & Unilateral & 45 (61.6) & $37(61.7)$ & $8(61.5)$ & 0.993 \\
\hline & Bilateral & $28(38.4)$ & $23(38.3)$ & $5(38.5)$ & \\
\hline \multirow[t]{2}{*}{ CXR extension lobes } & Localized & $29(39.7)$ & $24(40.0)$ & $5(38.5)$ & 0.918 \\
\hline & Multi-lobar & $44(60.3)$ & $36(60.0)$ & $8(61.5)$ & \\
\hline \multirow[t]{2}{*}{ CXR cavity } & Present & $41(56.2)$ & $30(50.0)$ & $11(84.6)^{*}$ & 0.031 \\
\hline & Absent & $32(43.8)$ & $30(50.0)$ & $2(15.4)$ & \\
\hline
\end{tabular}

*Shows significantly higher proportion at $5 \%$ level of significance by using chi-square test

Values given in parentheses are percentages

${ }^{1}$ Sig significance

${ }^{2} C X R$ chest radiograph
Table 3 Predicting factors of sputum smear positivity

\begin{tabular}{lllll}
\hline Factors & $\begin{array}{l}\text { Sputum converted } \\
(n=60)\end{array}$ & $\begin{array}{l}\text { Sputum non-con- } \\
\text { verted }(n=13)\end{array}$ & OR $^{1}\left(95 \% \mathrm{CI}^{2}\right)$ & $p$-value \\
\hline Male patients & $36(60.0)$ & $7(53.8)$ & $0.78(0.23-2.60)$ & 0.683 \\
Age $>40$ years & $32(53.3)$ & $6(46.2)$ & $1.33(0.40-4.44)$ & 0.639 \\
$\mathrm{BMI}^{3}<18.5 \mathrm{~kg} / \mathrm{m}^{2}$ & $22(37.3)$ & $5(38.5)$ & $1.09(0.28-4.28)$ & 0.901 \\
Employed & $21(35.0)$ & $7(53.8)$ & $1.83(0.47-7.19)$ & 0.385 \\
Illiterate & $24(40.0)$ & $6(46.2)$ & $1.29(0.38-4.30)$ & 0.683 \\
Married & $40(66.7)$ & $10(76.9)$ & $1.67(0.41-6.74)$ & 0.474 \\
Lower economic class & $26(43.3)$ & $6(46.2)$ & $1.12(0.34-3.74)$ & 0.853 \\
Own house & $35(58.3)$ & $8(61.5)$ & $1.14(0.33-3.91)$ & 0.831 \\
Smoking & $24(40.0)$ & $4(30.8)$ & $0.67(0.18-2.41)$ & 0.537 \\
Diabetes & $14(23.3)$ & $3(23.1)$ & $0.99(0.24-4.09)$ & 0.984 \\
$3+$ smear & $24(40.0)$ & $6(46.2)$ & $1.29(0.38-4.30)$ & 0.683 \\
CXR ${ }^{4}$ bilateral sides & $23(38.3)$ & $5(38.5)$ & $0.73(0.21-2.56)$ & 0.627 \\
CXR multi-lobe extension & $36(60.0)$ & $8(61.5)$ & $1.07(0.31-3.65)$ & 0.918 \\
CXR cavity & $\underline{30(50.0)}$ & $\underline{11(84.6) *}$ & $\underline{5.50(1.12-27.0)}$ & $\underline{0.035}$ \\
\hline
\end{tabular}

* Shows significantly higher proportion at $5 \%$ level of significance

${ }^{1} \mathrm{OR}$ odds ratio

${ }^{2} \mathrm{CI}$ confidence interval

${ }^{3} \mathrm{BMI}$ body mass index

${ }^{4} C X R$ chest radiograph 
load (smear grade $3+$ ) and likelihood of persistent sputum positivity, though our study has shown no such association $(p=0.683$, OR $1.29,95 \%$ CI $0.38-4.30)$.

Radiologic extent of disease has been reported by several studies as being a predictor of sputum non-conversion [10, $12,23]$. This includes the involvement of a greater number of lobes, cavitation, and/or military disease. We investigated the extent (unilateral/bilateral and multilobar) of disease but found no association with persistent sputum positivity. However, the presence of one or more cavities $(\mathrm{OR}=5.50, \mathrm{CI}$ $1.12-27.0 ; p=0.035$ ) strongly predicted non-conversion of sputum at 2 months. Our finding is corroborated by similar reports from other studies [3, 11, 22, 23] that have also documented cavitation as a predictor of persistent sputum positivity. Interestingly, half of our patients who did convert also had one or more cavities on the CXR.

A limitation of the present study is the use of smear microscopy for follow-up (albeit in accordance with WHO guidelines [20] and the local TB Control Programme [19]) at 2 months. Ideally, it is the negative cultures that truly reflect sputum conversion but getting cultures is less cost-effective and more time-consuming and therefore not feasible. Since the microscopy also reports non-viable bacteria, its correlation with culture (which is the gold standard) is debatable; a German study [27] has reported a strong correlation between smear microcopy and time to culture positivity within the first 2 months of treatment. The same holds true for Xpert MTB/RIF, a rapid, easily available nucleic acid amplification test; its ability to detect non-viable bacilli with greater sensitivity precludes its use as a follow-up tool.

An understanding of the relevance of these factors pertaining to persistent sputum positivity can help clinicians foresee which patients will remain infectious for a longer period of time and may have worse outcomes. This stratification may also improve large-scale TB control activities. The current study sets a precedent for future much larger studies at national level that could also draw conclusions about outcomes at the end of treatment and occurrence of relapse. Previous studies have highlighted the association between high smear grades and treatment failure/relapse [26-28].

\section{Conclusion}

Our study shows that the presence of one or more cavities can strongly predict ( $p=0.035$ ) sputum non-conversion at 2 months and thus flag patients who may be at risk for poor outcomes. One of the key measures in making TB treatment and control activities a success is identifying patients at high risk of transmission and poor outcomes. The recognition of individuals at high risk of non-conversion through the use of chest radiograph (which is done across all TB centers, even in low-income countries) would help national TB control programs focus their resources (strict monitoring and DOT) towards care for these particular patients.

Supplementary Information The online version contains supplementary material available at https://doi.org/10.1007/s42399-021-01098-6.

Author Contribution Nadia Jawad: Acquisition and analysis of data and drafting the work.

Saira Jafri: Analysis and interpretation of data and revising the manuscript critically for important intellectual content.

Nausheen Saifullah: Final approval of the version to be published. Naseem Ahmed: Revising the work critically and supervision.

\section{Availability of Data and Material N/A.}

Code Availability N/A.

\section{Declarations}

Ethics Approval Taken from JPMC Institutional Review Board Committee on June 10, 2019.

Consent to Participate An informed consent for participation was taken from either the patient themselves or the attendant, whichever was possible.

Consent for Publication N/A.

Conflict of Interest The authors declare no competing interests.

\section{References}

1. World Health Organization. Tuberculosis Factsheets. March 2020. Available from https://www.who.int/news-room/fact-sheets/detail/ tuberculosis.

2. Parikh R, Nataraj G, Kanade S, Khatri V, Mehta P. Time to sputum conversion in smear positive pulmonary TB patients on category I DOTS and factors delaying it. J Assoc Physicians India. 2012 Aug;60(8):22-6.

3. Singla R, Osman MM, Khan N, Al-Sharif N, Al-Sayegh MO, Shaikh MA. Factors predicting persistent sputum smear positivity among pulmonary tuberculosis patients 2 months after treatment. Int J Tuberc Lung Dis. 2003 Jan 1;7(1):58-64.

4. Tiwari S, Kumar A, Kapoor SK. Relationship between sputum smear grading and smear conversion rate and treatment outcome in the patients of pulmonary tuberculosis undergoing dots-a prospective cohort study. Indian J Tuberc. 2012 Jul;59(3):135-40.

5. Vora SD, Gandhi RV, Vasava SA, Ganava AR, Amin G. A study of factors affecting sputum conversion in patients of pulmonary tuberculosis. Natl J Community Med. 2016; 7(2): 142-146. http:// www.njcmindia.org/home/download/792.

6. D'Souza KA, Zaidi SM, Jaswal M, Butt S, Khowaja S, Habib SS, et al. Factors associated with month 2 smear non-conversion among category 1 tuberculosis patients in Karachi, Pakistan. J Infect Public Health. 2018 Mar 1;11(2):283-5. https://doi.org/10. 1016/j.jiph.2017.06.009.

7. Horne DJ, Royce SE, Gooze L, Narita M, Hopewell PC, Nahid $\mathrm{P}$, et al. Sputum monitoring during tuberculosis treatment for predicting outcome: systematic review and meta-analysis. Lancet Infect Dis. 2010 Jun 1;10(6):387-94. https://doi.org/10.1016/ S1473-3099(10)70071-2. 
8. Fortún J, Martín-Dávila P, Molina A, Navas E, Hermida JM, Cobo $\mathrm{J}$, et al. Sputum conversion among patients with pulmonary tuberculosis: are there implications for removal of respiratory isolation? J Antimicrob Chemother. 2007 Apr 1;59(4):794-8. https://doi.org/ $10.1093 / \mathrm{jac} / \mathrm{dkm} 025$.

9. Unsematham S, Kateruttanakul P. Factors predicting sputum smear conversion and treatment outcomes in new smear-positive pulmonary tuberculosis. J Med Assoc Thailand= Chotmaihet thangphaet. 2013;96(6):644. https://pubmed.ncbi.nlm.nih.gov/ $23951819 /$

10. Bouti K, Aharmim M, Marc K, Soualhi M, Zahraoui R, Benamor $\mathrm{J}$, et al. Factors influencing sputum conversion among smear-positive pulmonary tuberculosis patients in Morocco. ISRN Pulmonol. 2013 Jun;27:2013. https://doi.org/10.1155/2013/486507.

11. Dominguez-Castellano A, Muniain MA, Rodriguez-Bano J, Garcia M, Rios MJ, Galvez J, Perez-Cano R. Factors associated with time to sputum smear conversion in active pulmonary tuberculosis. The Int J Tuberc Lung Dis. 2003;7(5):432-8. https://www. ingentaconnect.com/content/iuatld/ijtld/2003/00000007/00000 005/art00007.

12. Babalik A, Kiziltas S, Arda H, Oruc K, Celintas G, Calalettin H. Factors affecting smear conversion in tuberculosis management. Med Sci. 2012;1(4):351-62. https://doi.org/10.5455/medscience. 2012.01.8036.

13. Moderbacher CR, Ramirez SI, Dan JM, Grifoni A, Hastie KM, Weiskopf D, Belanger S, Abbott RK, Kim C, Choi J, Kato Y. Antigen-specific adaptive immunity to SARS-CoV-2 in acute COVID-19 and associations with age and disease severity. Cell. 2020 Nov 12;183(4):996-1012.

14. Sheerin D, Abhimanyu XW, Johnson WE, Coussens A. Systematic evaluation of transcriptomic disease risk and diagnostic biomarker overlap between COVID-19 and tuberculosis: a patient-level meta-analysis. medRxiv. 2020.

15. Tadolini M, Codecasa LR, García-García JM, Blanc FX, Borisov $\mathrm{S}$, Alffenaar JW, et al. Active tuberculosis, sequelae and COVID19 co-infection: first cohort of 49 cases. Eur Respir J. 2020;56(1).

16. TB/COVID-19 Global Study Group. TB and COVID-19 co-infection: rationale and aims of a global study. Int J Tuberc Lung Dis. 2021;25(1). https://doi.org/10.5588/ijtld.20.0786.

17 Stochino C, Villa S, Zucchi P, Parravicini P, Gori A, Raviglione MC. Clinical characteristics of COVID-19 and active tuberculosis co-infection in an Italian reference hospital. Eur Respir J. 2020;56(1):2001708. https://doi.org/10.1183/13993003. 01708-2020.

18. Migliori GB, Thong PM, Akkerman O, Alffenaar JW, ÁlvarezNavascués F, Assao-Neino MM, et al. Worldwide effects of coronavirus disease pandemic on tuberculosis services, January--April 2020. Emerg Infect Dis. 2020;26(11):2709-12. https://doi.org/10. 3201/eid2611.203163.

19. National Tuberculosis Control Program. National guidelines for the control of tuberculosis in Pakistan (revised 2019). 2019.
Available from: http://ntp.gov.pk/ntp-old/uploads/National_Guide lines_for_TB_Revised_2019.pdf.

20. World Health Organization. WHO Guidelines for treatment of drug-susceptible tuberculosis and patient care (2017update). 2017. Available from: http://apps.who.int/iris/bitstream/10665/255052/ 1/9789241550000-eng.pdf?ua=1.

21. World Health Organization. Mycobacteriology Laboratory Manual. April 2014. Available from: https://www.who.int/tb/labor atory/mycobacteriology-laboratory-manual.pdf.

22. Wang JY, Lee LN, Yu CJ, Chien YJ, Yang PC, Tami Group. Factors influencing time to smear conversion in patients with smearpositive pulmonary tuberculosis. Respirology. 14(7):1012-9. https://doi.org/10.1111/j.1440-1843.2009.01598.x.

23. Diktanas S, Vasiliauskiene E, Polubenko K, Danila E, Celedinaite I, Boreikaite E, et al. Factors associated with persistent sputum positivity at the end of the second month of tuberculosis treatment in Lithuania. Tuberc Respir Dis. 2018;81(3):233-40. https://doi. org/10.4046/trd.2017.0096.

24. Yellappa V, Kandpal V, Lall D, Tabassum A. Determinants of sputum conversion at two months of treatment under National Tuberculosis Programme, South India. Int J Med Sci Public Health. 20165(12):1. https://www.academia.edu/download/48328166/ Determinants_of_sputum_conversion.pdf.

25. Mota PC, Carvalho A, Valente I, Braga R, Duarte R. Predictors of delayed sputum smear and culture conversion among a Portuguese population with pulmonary tuberculosis. Revista Portuguesa de Pneumologia (English Edition). 2012 Mar 1;18(2):72-9. https:// doi.org/10.1016/j.rppnen.2012.01.004.

26. Nwokeukwu HI, Awujo DN, Emma-Ukeagbu U. Association of sputum conversion and outcome with initial smear grading among new smear positive Tuberculosis patients in a Tertiary Health Facility, South East Zone, Nigeria. J Dent Med Sci. 2013;4(6):2279-0853. http://iosrjournals.org/iosr-jdms/papers/ Vol4-issue6/B0460409.pdf.

27. Olaru ID, Heyckendorf J, Grossmann S, Lange C. Time to culture positivity and sputum smear microscopy during tuberculosis therapy. PLoS One. 2014;9(8):e106075. https://pdfs.semanticsc holar.org/0091/55c8ac859386aa41cd86b7935abf2382dddd.pdf.

28. Hesseling AC, Walzl G, Enarson DA, Carroll NM, Duncan K, Lukey PT et al. Baseline sputum time to detection predicts month two culture conversion and relapse in non-HIV-infected patients. The international journal of tuberculosis and lung disease. 2010;14(5):560-70. https://pubmed.ncbi.nlm.nih.gov/20392348/.

Publisher's Note Springer Nature remains neutral with regard to jurisdictional claims in published maps and institutional affiliations. 